\title{
Sublobar resection is equivalent to lobectomy for clinical stage 1A lung cancer in solid nodules
}

\author{
Nasser K. Altorki, MD, ${ }^{\mathrm{a}}$ Rowena Yip, MPH, ${ }^{\mathrm{b}}$ Takaomi Hanaoka, MD, ${ }^{\mathrm{c}}$ Thomas Bauer, MD, ${ }^{\mathrm{d}}$ \\ Ralph Aye, MD, ${ }^{\mathrm{e}}$ Leslie Kohman, MD, ${ }^{\mathrm{f}}$ Barry Sheppard, MD, ${ }^{\mathrm{g}}$ Richard Thurer, MD, ${ }^{\mathrm{h}}$ \\ Shahriyour Andaz, MD, ${ }^{\mathrm{i}}$ Michael Smith, MD ${ }^{\mathrm{j}}$ William Mayfield, MD, ${ }^{\mathrm{k}}$ Fred Grannis, MD, ${ }^{1}$ \\ Robert Korst, MD ${ }^{\mathrm{m}}$ Harvey Pass, MD,${ }^{\mathrm{n}}$ Michaela Straznicka, MD, ${ }^{\circ}$ Raja Flores, MD, ${ }^{\mathrm{b}}$ and \\ Claudia I. Henschke, $\mathrm{PhD}, \mathrm{MD},{ }^{\mathrm{b}}$ for the I-ELCAP Investigators
}

Objectives: A single randomized trial established lobectomy as the standard of care for the surgical treatment of early-stage non-small cell lung cancer. Recent advances in imaging/staging modalities and detection of smaller tumors have once again rekindled interest in sublobar resection for early-stage disease. The objective of this study was to compare lung cancer survival in patients with non-small cell lung cancer with a diameter of 30 $\mathrm{mm}$ or less with clinical stage 1 disease who underwent lobectomy or sublobar resection.

\begin{abstract}
Methods: We identified 347 patients diagnosed with lung cancer who underwent lobectomy $(\mathrm{n}=294)$ or sublobar resection $(\mathrm{n}=53)$ for non-small cell lung cancer manifesting as a solid nodule in the International Early Lung Cancer Action Program from 1993 to 2011. Differences in the distribution of the presurgical covariates between sublobar resection and lobectomy were assessed using unadjusted $P$ values determined by logistic regression analysis. Propensity scoring was performed using the same covariates. Differences in the distribution of the same covariates between sublobar resection and lobectomy were assessed using adjusted $P$ values determined by logistic regression analysis with adjustment for the propensity scores. Lung cancer-specific survival was determined by the Kaplan-Meier method. Cox survival regression analysis was used to compare sublobar resection with lobectomy, adjusted for the propensity scores, surgical, and pathology findings, when adjusted and stratified by propensity quintiles.
\end{abstract}

Results: Among 347 patients, 10-year Kaplan-Meier for 53 patients treated by sublobar resection compared with 294 patients treated by lobectomy was $85 \%$ (95\% confidence interval, $80-91$ ) versus $86 \%$ (confidence interval, 75-96) $(P=.86)$. Cox survival analysis showed no significant difference between sublobar resection and lobectomy when adjusted for propensity scores or when using propensity quintiles $(P=.62$ and $P=.79$, respectively). For those with cancers $20 \mathrm{~mm}$ or less in diameter, the 10 -year rates were $88 \%(95 \%$ confidence interval, $82-93)$ versus $84 \%$ (95\% confidence interval, 73-96) $(P=.45)$, and Cox survival analysis showed no significant difference between sublobar resection and lobectomy using either approach $(P=.42$ and $P=.52$, respectively).

Conclusions: Sublobar resection and lobectomy have equivalent survival for patients with clinical stage IA nonsmall cell lung cancer in the context of computed tomography screening for lung cancer. (J Thorac Cardiovasc Surg 2014;147:754-64)

\footnotetext{
From the NY Presbyterian Hospital/Weill Cornell Medical College, ${ }^{a}$ New York, NY; Icahn School of Medicine at Mount Sinai, ${ }^{b}$ New York, NY; Azumi General Hospital, ${ }^{\mathrm{c}}$ Nagano, Japan; Helen F. Graham Cancer Center, ${ }^{\mathrm{d}}$ Newark, Del; Swedish Med-

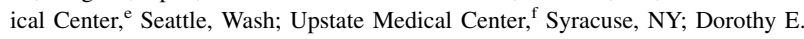
Schneider Cancer Center, ${ }^{g}$ Mills-Peninsula Health Services, San Mateo, Calif; Jackson Memorial Hospital, ${ }^{\text {h }}$ University of Miami, Miami, Fla; South Nassau Communities Hospital, ${ }^{\mathrm{i}}$ Long Island, NY; Georgia Institute for Lung Cancer Research, ${ }^{\mathrm{j}}$ Atlanta, Ga; Wellstar Health System, ${ }^{\mathrm{k}}$ Marietta, Ga; City of Hope National Medical Center, ${ }^{1}$ Duarte, Calif; The Valley Hospital Cancer Center, ${ }^{\mathrm{m}}$ Paramus, NJ; New York University Medical Center, ${ }^{\mathrm{n}}$ New York, NY; and John Muir Cancer Institute, ${ }^{\circ}$ Concord, Calif.

The I-ELCAP pooled database has been supported in part by National Institutes of Health R01-CA-633931 and R01-CA-78905; Department of Energy DE-FG0296SF21260; The City of New York, Department of Health and Mental Hygiene; New York State Office of Science, Technology and Academic Research; American Cancer Society; Israel Cancer Association; The Starr Foundation; The New York Community Trust; The Rogers Family Fund; The Foundation for Lung Cancer: Early Detection, Prevention, and Treatment (primary source was an unrestricted gift in 2000-2003 from the Vector Group, the parent company of Liggett Tobacco); Dorothy R. Cohen Foundation, Research Foundation of Clinic Hirslanden; YadHanadiv Foundation; Jacob and Malka Goldfarb Charitable Foundation;
}

\footnotetext{
Auen/Berger Foundation; Princess Margaret Foundation; Berger Foundation; Mills Peninsula Hospital Foundation, Tenet Healthcare Foundation; Ernest E. Stempel Foundation; Academic Medical Development Corporation; Columbia University Medical Center, Empire Blue Cross and Blue Shield; Eastman-Kodak Corporation; General Electric Corporation; Weill Medical College of Cornell University; Cornell University; New York Presbyterian Hospital; Clinic Hirslanden; Swedish Hospital; Christiana Care Helen F. Graham Cancer Center; Holy Cross Hospital; Eisenhower Hospital; Jackson Memorial Hospital Health System; and Evanston Northwestern Healthcare.

Disclosures: Authors have nothing to disclose with regard to commercial support.

Read at the 93rd Annual Meeting of The American Association for Thoracic Surgery, Minneapolis, Minnesota, May 4-8, 2013.

The I-ELCAP Investigators are listed in Appendix 1.

Received for publication May 8, 2013; revisions received July 30, 2013; accepted for publication Sept 30, 2013; available ahead of print Nov 25, 2013

Address for reprints: Claudia I. Henschke, PhD, MD, Department of Radiology, Mount Sinai School of Medicine, 1 Gustave Levy Place, Box 1234, New York, NY (E-mail: Claudia.Henschke@mountsinai.org).

0022-5223/\$36.00

Copyright () 2014 by The American Association for Thoracic Surgery

http://dx.doi.org/10.1016/j.jtcvs.2013.09.065
} 


\section{Abbreviations and Acronyms \\ CAC $=$ coronary artery calcification \\ $\mathrm{CI}=$ confidence interval \\ CT $=$ computed tomography \\ HR = hazard ratio \\ I-ELCAP $=$ International Early Lung Cancer Action Program \\ LCSG = Lung Cancer Study Group \\ $\mathrm{LR}=$ lobar resection \\ NSCLC $=$ non-small cell lung cancer \\ PET = positron emission tomography \\ SLR = sublobar resection}

The results of the randomized trial reported by the Lung Cancer Study Group (LCSG) in 1995 established lobectomy as the standard of surgical care for stage I non-small cell lung cancer (NSCLC) in patients who are able to tolerate the procedure. ${ }^{1}$ In that trial, conducted throughout the 1980s, 276 patients with clinical stage I (cT1N0M0) NSCLC detected on plain chest radiography were randomly assigned to lobar resection (LR) or sublobar resection (SLR) that included wedge resection or anatomic segmentectomy. LR was associated with a lower rate of local recurrence and lung cancer-related deaths.

Important advances in clinical staging modalities and the ability to detect smaller tumors by computed tomography (CT) scans have led to a resurgence of interest in SLR for early-stage lung cancer. ${ }^{2-12}$ Theoretic advantages of SLR include preservation of lung parenchyma, pulmonary function, and lower perioperative morbidity, thus enhancing the possibilities of future resections for additional primary lung cancers. The significant proportion of patients with early-stage lung cancer who are not currently candidates for LR because of impaired pulmonary function may be safely and effectively treated by SLR provided that the survival outcome is equivalent to that of LR. Several institutional retrospective case series have suggested that recurrence and survival may be similar after both types of resection. ${ }^{7-12}$ An analysis from the large National Cancer Institute registry of 1165 patients with lung cancer with tumors $2 \mathrm{~cm}$ or less showed that survival of 969 patients undergoing LR was not significantly different from the 196 patients undergoing SLR, even when accounting for covariates that might be confounders (eg, age and comorbidities). ${ }^{13,14}$

The primary objective of the current work is to compare the survival of patients with clinical stage I A NSCLC (cT1N0M0) who were treated with LR or SLR in the International Early Lung Cancer Action Program (I-ELCAP). I-ELCAP is an international study that prospectively enrolls all individuals from participating screening programs that follow a common protocol that provides for data pool- ing. ${ }^{15,16}$ At the time of enrollment, participants are asymptomatic for lung cancer, and pertinent baseline demographic and clinical information are collected. CT findings at baseline or annual repeat rounds of screening are documented together with any subsequent diagnosis of lung cancer, treatment, and follow-up. This large resource offers a unique platform to examine the extent of surgical resection for early-stage lung cancer diagnosed under screening and provides the opportunity to further address possible confounders of the decision to perform LR or SLR.

\section{MATERIALS AND METHODS}

The I-ELCAP database was queried for all patients diagnosed with a first primary clinical stage I NSCLC (cT1NOM0) as a result of baseline and subsequent annual CT screening from 1993 to 2011. The screenings were performed according to a common protocol that specified the initial CT test, the definition of positive results, and the recommended diagnostic workup. $^{15,16}$ The protocol required that all subjects be asymptomatic and deemed by their physicians as suitable candidates for surgical resection in the event that a diagnosis of lung cancer was made, but the enrollment criteria as to age and smoking status was decided by each participating institution. The extent of surgery, including the extent of mediastinal lymph node sampling, was not mandated by the protocol but was according to the standard of care at each institution. All screenees gave informed consent for baseline and repeat screenings under institutional review board-approved protocols at each of the participating institutions.

For the purposes of the current study, only patients whose lung cancer manifested as a solid nodule on the CT scan were included. This classification was performed by an experienced chest radiologist at the I-ELCAP Coordinating Center. The data extracted for analysis included demographic information, smoking history, and the self-reporting of the presence or absence of 21 preexisting conditions. These 21 preexisting comorbidities were categorized into 6 categories (cardiac diseases, vascular disease, chronic obstructive pulmonary disease, diabetes, other non-lung cancers, other diseases). The presence of each of these preexisting conditions was coded as 1 ( 0 otherwise), and the sum was used as the cumulative comorbidity score.

Imaging information on the baseline $\mathrm{CT}$ scan included the presence and extent of emphysema and coronary artery calcification (CAC). We previously reported that screening participants with marked emphysema were found to be at increased risk of dying of emphysema and lung cancer, and those with a CAC score of 4 to 12 had an increased risk of dying of coronary artery disease. ${ }^{17-21}$

Once lung cancer was diagnosed, peripheral location of the tumor, clinical staging, details of surgical treatment, results of pathologic examination, and survival status were documented. Although preoperative pulmonary function test results were not initially documented in the I-ELCAP database, the presence and extent of emphysema on the baseline CT scan were available on all patients and have been shown to be a surrogate indicator of lung function. ${ }^{20}$ Tumor location was classified as peripheral if it was within $2 \mathrm{~cm}$ of the costal pleura and otherwise as central. The clinical stage of each patient was determined and entered into the database at the time of initial diagnosis. Tumor diameter was the average of the length and width of the tumor on the CT image that showed the largest crosssection of the nodule before resection. The presence or absence of lymph-node and distant metastases ( $\mathrm{N}$ and $\mathrm{M}$ status) was assessed in the most recent CT scan before diagnosis and from a positron emission tomography (PET) scan, if performed. Nodal status was classified as N0 if the widths of all mediastinal lymph nodes were less than $10 \mathrm{~mm}$, no hilar lymph nodes were identified, and the PET scan, if done, showed no 
abnormal uptake. Resections for lung cancer were performed by dedicated general thoracic surgeons.

Each case was reviewed to determine the extent of resection and if treated by SLR or LR, the former including by segmentectomy or wedge resections. The number of nodal stations sampled or dissected at the time of surgery also was documented. The surgical specimens were examined at each institution according to the I-ELCAP pathology protocol. ${ }^{22}$ Histologic slides of the tumors were sent to the Coordinating Center for review and consensus diagnosis by a 5-member Pathology Review Panel following the protocol. ${ }^{23,24} \mathrm{~A}$ member of the panel also reviewed all the slides to identify pleural, lymphatic, and vascular invasion. Cell-type classification for purposes of this report was adenocarcinoma, squamous cell carcinoma, large-cell carcinoma, and other carcinoma (atypical carcinoid, sarcoma, spindle cell/pleomorphic, non-small cell unspecified) according to the World Health Organization criteria and subsequent reclassification of adenocarcinoma, bronchioloalveolar subtype into adenocarcinoma in situ. ${ }^{25,26}$

Recurrence and postoperative treatment were documented by each participating institution. Recurrence was not further classified to local regional or systemic. All patients were followed to death from lung cancer, last contact, or April 30, 2013. Follow-up time ranged from 1 to 209 months, and median follow-up time was determined. All deaths from lung cancer were recorded, including treatment-related mortality. At each I-ELCAP participating institution, the time and cause of death were ascertained in every case by direct family or referring physician contact and recorded in the database.

\section{Statistical Analysis}

Differences in the distribution of demographic, clinical characteristics, and comorbidity score for patients undergoing SLR or LR were evaluated using logistic regression analysis based on the baseline characteristics, findings on baseline CT scan, and presurgical CT scan to determine the unadjusted $P$ values (Table 1). To ensure a balance of the covariates between the 2 surgical treatments because there was nonrandom allocation to each creating potential bias, propensity scoring was used as has been for other similar analyses. ${ }^{15,16,23,24}$ The propensity score was calculated for each patient using logistic regression analysis ( 1 assigned to SLR and 0 to LR) based on each patient's baseline characteristics, findings on baseline, and presurgical CT scans (Table 1). This score represents the probability of being assigned to 1 of the 2 types of surgery, SLR or LR, conditional on all the covariates included in the propensity score determination. The regression analysis was then performed again to compare SLR with LR for each covariate with the comparison being adjusted for the propensity score (adjusted $P$ value, Table 1 ).

Lung cancer-specific survival was determined by the Kaplan-Meier method. Ten-year Kaplan-Meier rates were determined for patients in each of the 2 treatment groups, LR or SLR, for all cases, and those cases with a diameter was $20 \mathrm{~mm}$ or less. Log-rank test was used to assess differences in the survival of the 2 treatment groups.

The multivariate Cox survival analysis was performed in 2 ways. First, the SLR comparison with LR was performed for all patients adjusted by the propensity score and surgical and pathology findings (Table 2) not included in the propensity score determination and then again, when stratified by the propensity score quintiles. The same analysis is repeated for all those with clinical stage IA disease for whom the presurgical tumor size was $20 \mathrm{~mm}$ or less. A secondary analysis was performed to compare SLR wedge resection with LR and SLR segmentectomy with LR using Cox regression analysis as detailed earlier. All analyses were performed using SAS version 9.2 software (SAS Institute Inc, Cary, NC).

\section{RESULTS}

NSCLC manifested as a solid nodule in 347 patients who are the subject of this report. Lobectomy was performed in
294 patients, and SLR was performed in 53 patients ( 16 by segmentectomy and 37 by wedge resection). The baseline and presurgical covariates for the 2 groups are shown in Table 1 . The majority of patients were current or former smokers of white ethnicity. Although there were fewer current smokers in the SLR group than in the LR group (51\% vs $54 \%, P=.27$ ), the median pack-years of smoking was slightly higher in the SLR group (50 vs $48, P=.74$ ), but neither were significant. None of the self-reported comorbidities and the cumulative morbidity score were significantly different, although SLR had more patients who reported cardiac disease $(11 \%$ vs $6 \%, P=.14)$ and chronic obstructive pulmonary disease ( $26 \%$ vs $15 \%, P=.05$ ). On the baseline CT scan, the frequencies of CAC $(51 \%$ vs $52 \%, P=.95)$, emphysema ( $47 \%$ vs $48 \%, P=.95)$, and marked emphysema ( $4 \%$ vs $4 \%, P=.85$ ) were essentially identical for SLR and LR. The CAC score of 4 to $12(26 \%$ vs $18 \%, P=.18$ ), suggestive of coronary artery disease, was more frequent in SLR than in LR, but these were not significant. A total of 142 PET scans were performed; of the $22(37 \%)$ of the 53 patients treated by SLR and 120 $(42 \%)$ of the 294 patients treated by LR, the 2 proportions were not significantly different $(P=.92)$. After adjusting for the propensity score, none of the covariates included in creating the score were significant as shown by the adjusted $P$ values in Table 1 .

Pathologic stage I disease was confirmed in $92 \%$ $(\mathrm{n}=49)$ of the 53 patients treated by SLR and in $86 \%$ $(\mathrm{n}=252)$ of the 294 patients treated by LR. Patients treated by SLR had mediastinal lymph node resection less frequently than those treated by LR $(55 \%$ vs $79 \%$, $P=.0003$ ) (Table 2). Among those who underwent mediastinal lymph node resection, the pathologic upstaging to mediastinal disease (N2 and N3) occurred in 7\% (2/29) of patients with SLR and 4\% (9/231) of patients with LR. No significant differences were found between SLR and LR for the pathology findings. Postsurgical treatment, if any, was not significantly different $(11 \%$ vs $13 \%$, $P=.75$ ). There were no postsurgical deaths (within 1 month of surgery) in the patients with SLR and in 3 patients with LR. Lung-cancer specific death, including any cause of death within 1 month of surgery, occurred in $11 \%(6 / 53)$ and $11 \%(32 / 294)$ of patients with SLR and LR, respectively, and death from all causes occurred in $23 \%(12 / 53)$ and $24 \%(70 / 294)$ of patients, respectively. The recurrence rate in the SLR group was higher, but not significantly, than in the LR group $(19 \%$ vs $12 \%, P=.15)$. In the SLR group, all recurrences occurred in those who had wedge resection.

The asymptote of the 10-year Kaplan-Meier survival curve by SLR and LR was not significantly different, $86 \%(95 \%$ confidence interval $[\mathrm{CI}], 75-96)$ versus $85 \%$ (95\% CI, 80-91) $(P=.86)$ (Figure 1). Overall median survival time was 65 months, 61 months for SLR and 66 
TABLE 1. Presurgical characteristics of 348 patients with clinical stage I lung cancer less than $30 \mathrm{~mm}$ in diameter

\begin{tabular}{|c|c|c|c|c|}
\hline \multirow[b]{2}{*}{ Extent of surgery } & \multicolumn{2}{|c|}{ Extent of resection } & \multicolumn{2}{|c|}{$P$ value } \\
\hline & Lobectomy & Sublobar resection & Unadjusted & Adjusted* $^{*}$ \\
\hline No. of cases & 294 & 53 & & \\
\hline \multicolumn{5}{|l|}{ Baseline characteristics } \\
\hline Male gender & $152(52 \%)$ & $29(55 \%)$ & .69 & .91 \\
\hline Median age (IQR) & $63(59-68)$ & $65(59-72)$ & .15 & .95 \\
\hline $40-49$ y & $9(3 \%)$ & $1(2 \%)$ & & \\
\hline $50-59$ y & $65(22 \%)$ & $13(25 \%)$ & & \\
\hline $60-69$ y & $158(54 \%)$ & $25(47 \%)$ & & \\
\hline $70+y$ & $62(21 \%)$ & $14(26 \%)$ & & \\
\hline White ethnicity & $253(86 \%)$ & $44(83 \%)$ & .56 & .99 \\
\hline College education & $103(35 \%)$ & $19(36 \%)$ & .91 & .99 \\
\hline \multicolumn{5}{|l|}{ Smoking history } \\
\hline Never smoker & $12(4 \%)$ & $4(8 \%)$ & Ref & Ref \\
\hline Former smoker & $122(41 \%)$ & $22(42 \%)$ & .32 & .87 \\
\hline Current smoker & $160(54 \%)$ & $27(51 \%)$ & .27 & .83 \\
\hline Median pack-years (IQR) & $48(35-68)$ & $50(38-70)$ & .74 & .96 \\
\hline \multicolumn{5}{|l|}{ Comorbidities } \\
\hline Cardiac & $17(6 \%)$ & $6(11 \%)$ & .14 & .84 \\
\hline Vascular disease & $62(21 \%)$ & $12(23 \%)$ & .80 & .93 \\
\hline COPD & $45(15 \%)$ & $14(26 \%)$ & .05 & .96 \\
\hline Diabetes & $25(9 \%)$ & $4(8 \%)$ & .82 & 1.00 \\
\hline Other cancer & $40(14 \%)$ & $7(13 \%)$ & .94 & .95 \\
\hline Other diseases & $58(20 \%)$ & $10(19 \%)$ & .88 & .88 \\
\hline Median comorbidity score (IQR) & $1(0-1)$ & $1(0-2)$ & .49 & $\dagger$ \\
\hline \multicolumn{5}{|l|}{ Findings on baseline $\mathrm{CT}$} \\
\hline \multicolumn{5}{|l|}{ Emphysema } \\
\hline Any & $140(48 \%)$ & $25(47 \%)$ & .95 & $\dagger$ \\
\hline Marked & $13(4 \%)$ & $2(4 \%)$ & .83 & .98 \\
\hline \multicolumn{5}{|l|}{ Coronary calcification } \\
\hline Any & $154(52 \%)$ & $27(51 \%)$ & .85 & $\dagger$ \\
\hline CAC score $4-12$ & $54(18 \%)$ & $14(26 \%)$ & .18 & .88 \\
\hline \multicolumn{5}{|l|}{ Findings on $\mathrm{CT}$ before surgery } \\
\hline Peripheral location on CT & $205(69 \%)$ & $38(74 \%)$ & .77 & .93 \\
\hline \multicolumn{5}{|l|}{ Nodule diameter on $\mathrm{CT}$} \\
\hline$\leq 20 \mathrm{~mm}$ & $256(87 \%)$ & $50(94 \%)$ & .14 & .89 \\
\hline $21-30 \mathrm{~mm}$ & $38(13 \%)$ & $3(6 \%)$ & & \\
\hline
\end{tabular}

$C A C$, Coronary artery calcification; $C O P D$, chronic obstructive pulmonary disease; $C T$, computed tomography; IQR, interquartile range. *Adjusted for propensity score. $\dagger$ Not included in propensity score model.

months for LR. Cox survival analysis, after adjustment for the propensity score and postsurgical and pathology findings, resulted in a hazard ratio (HR) of 1.3 (95\% CI, 0.5$3.3 ; P=.62$; Table 3 ). When stratified by the propensity score quintiles, the Cox survival analysis resulted in an HR of 1.1 (95\% CI, 0.4-2.9; $P=.79)$. In both of these Cox survival analyses, only $\mathrm{T} 1$ increased survival time significantly compared with T2-T4 $(P=.003$ and $P=.003$, respectively).

By comparing only 306 patients whose tumor diameter before surgery was $20 \mathrm{~mm}$ or less, 256 were treated by LR and 50 were treated by SLR. Recurrence in the SLR group was higher, but not significantly, than in the LR group ( $20 \%$ vs $10 \%, P=.21)$. Mediastinal lymph node resection was performed less frequently in the
SLR group than in the LR group $(55 \%$ vs $80 \%$, $P=.0002)$. The 10 -year asymptote of the Kaplan-Meier survival for patients treated by SLR and LR was $88 \%$ (95\% CI, 82-93) versus $84 \%$ (95\% CI, 73-96), respectively, but was not significantly different $(P=.45)$ (Figure 2). Cox survival analysis, after adjustment for the propensity score and postsurgical and pathology findings, did not demonstrate a significant difference between SLR and LR (HR, 1.5; 95\% CI, 0.6-4.2; $P=.42$ ) (Table 3 ). When stratified by propensity score quintiles, there was no significant difference (HR, 1.4; 95\% CI, 0.5-3.8; $P=.52$ ). In both of these Cox survival analyses, only $\mathrm{T} 1$ increased survival time significantly compared with T2-T4 $(P=.004$ and $P=.005$, respectively). 
TABLE 2. Mediastinal lymph node resections and pathology findings of 348 patients with clinical stage I lung cancer less than $30 \mathrm{~mm}$ in diameter

\begin{tabular}{|c|c|c|c|c|}
\hline \multirow[b]{2}{*}{ Extent of surgery } & \multicolumn{2}{|c|}{ Extent of resection } & \multicolumn{2}{|c|}{$P$ value } \\
\hline & Lobectomy & Sublobar resection & Unadjusted & Adjusted* \\
\hline No. of cases & 294 & 53 & & \\
\hline \multicolumn{5}{|l|}{ Mediastinal lymph node resection } \\
\hline Any resected & $231(79 \%)$ & $29(55 \%)$ & .0003 & $\dagger$ \\
\hline Any metastases & $9(4 \%)$ & $2(7 \%)$ & .79 & $\dagger$ \\
\hline \multicolumn{5}{|l|}{ Pathologic findings } \\
\hline \multicolumn{5}{|l|}{ Carcinoma cell-type } \\
\hline Adenocarcinoma & $191(65 \%)$ & $36 \ddagger(68 \%)$ & Ref & $\dagger$ \\
\hline Squamous cell & $72(24 \%)$ & $11(21 \%)$ & .68 & \\
\hline Large cell & $25(9 \%)$ & $4(8 \%)$ & & \\
\hline Other & $6(2 \%)$ & $2(4 \%)$ & & \\
\hline \multicolumn{5}{|l|}{ Pathology tumor diameter } \\
\hline$\leq 20 \mathrm{~mm}$ & $239(81 \%)$ & $49(92 \%)$ & .07 & $\dagger$ \\
\hline$>20 \mathrm{~mm}$ & $55(19 \%)$ & $4(8 \%)$ & & \\
\hline \multicolumn{5}{|l|}{ Pathologic invasion } \\
\hline Pleura invasion & $80(27 \%)$ & $10(19 \%)$ & .21 & $\dagger$ \\
\hline Angiolymphatic invasion & $53(18 \%)$ & $6(11 \%)$ & .24 & $\dagger$ \\
\hline \multicolumn{5}{|l|}{ pT status } \\
\hline $\mathrm{T} 1: \leq 30 \mathrm{~mm}$ without pleura invasion & $197(67 \%)$ & $40(75 \%)$ & Ref & $\dagger$ \\
\hline $\mathrm{T} 2: \leq 30 \mathrm{~mm}$ and pleural invasion & $75(26 \%)$ & $10(19 \%)$ & .23 & \\
\hline $\mathrm{T} 2: \geq 30 \mathrm{~mm}$ without pleural invasion & $5(2 \%)$ & $1(2 \%)$ & & \\
\hline T3: additional $\mathrm{Ca}$ in same lobe & $16 \S(5 \%)$ & $0(0 \%)$ & & \\
\hline $\mathrm{T} 4$ : additional $\mathrm{Ca}$ in ipsilateral lobe & $1(0 \%)$ & $2(4 \%)$ & & \\
\hline \multicolumn{5}{|l|}{ pN status } \\
\hline No & $267(91 \%)$ & $51(96 \%)$ & Ref & $\dagger$ \\
\hline N1 & $18(6 \%)$ & $0(0 \%)$ & .21 & \\
\hline N2 & $8(3 \%)$ & $2(4 \%)$ & & \\
\hline N3 & $1(0 \%)$ & $0(0 \%)$ & & \\
\hline
\end{tabular}
invasion.

Within the SLR group, no recurrence occurred in those who underwent anatomic segmentectomy, but recurrence occurred in those who underwent wedge resection $(0 \%$ vs $27 \%, P=.95)$. The baseline or presurgical covariates were not significantly different between those who underwent segmentectomy compared with wedge resection. The asymptote of the Kaplan-Meier survival curve for SLR segmentectomy and SLR wedge was $100 \%$ and $80 \%(66 \%-95 \%)$, respectively $(P=.12)$. Cox survival analysis comparing SLR segmentectomy with LR, adjusted for the propensity scores and postsurgical and pathology findings, was not significantly different $(P=.99)$. SLR wedge compared with LR, whether adjusted for the propensity scores $(P=.19)$ or stratified by propensity quintiles $(P=.17)$, was not significantly different (Table 3$)$.

There was no significant difference in lung cancer survival between patients with lung cancers $30 \mathrm{~mm}$ or less in diameter when treated by LR or SLR, when considering potential confounders and different analyses.

\section{DISCUSSION}

The debate about the role of SLR in the treatment of earlystage lung cancer has occupied thoracic surgeons for decades. $^{2-12}$ The debate was partly resolved by the publication of the results of the randomized trial conducted by the LCSG showing that in comparison with LR, SLR was associated with a 3-fold increase in local recurrence and a $50 \%$ increase in cancer-related deaths. ${ }^{1} \mathrm{LR}$, whenever possible, has since become the preferred procedure.

Renewed interest in SLR has been fueled by several factors; foremost among them is that the LCSG trial was conducted in the 1980s on the basis of lung cancer detection by plain chest radiography and before the routine use of $\mathrm{CT}$ and more recently PET as standard staging modalities for lung cancer. Many of these patients possibly had undetected metastatic disease. There has also been a notable increase in the detection of smaller tumors than those included in the LCSG trial using recent generation CT scanners. With the reports of increased curability of lung cancer $^{27}$ and the publication of the National Lung Screening Trial showing that CT screening for lung cancer reduced the relative lung cancer mortality when compared with chest radiographic screening, ${ }^{28}$ the number of patients presenting with small tumors will continue to increase. Because more of these patients are cured of their first primary lung cancer, they are also likely to develop 


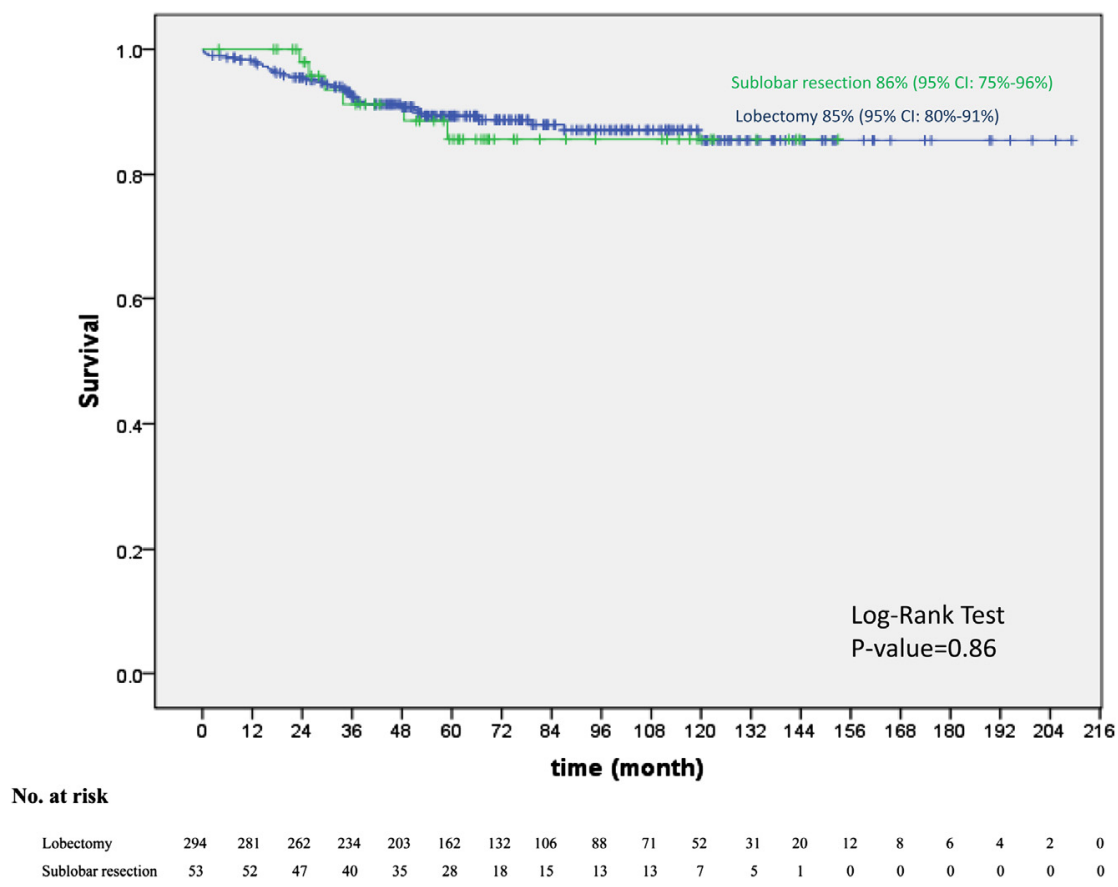

FIGURE 1. Kaplan-Meier survival curves for 337 patients with clinical stage IA lung cancer manifesting as a solid nodule (nodule diameter of $\leq 30 \mathrm{~mm}$ ), separately for those who underwent lobectomy $(\mathrm{n}=294)$ and SLR $(\mathrm{n}=53)$. CI, Confidence interval.

additional primaries and thus lung-sparing surgery will become increasingly important. There also has been a steady increase in the proportion of elderly patients presenting with incidentally detected, potentially curable early-stage disease, and in these patients SLR may be associated with lower morbidity and equivalent survival to lobectomy. ${ }^{29}$

The principal finding of this report is that SLR had equivalent survival outcome as LR in patients with screendetected clinical stage IA lung cancers manifesting as a

TABLE 3. Cox regression analysis for sublobar resection compared with lobectomy with the hazard ratio, adjusted for propensity scores and surgical and pathology covariates

\begin{tabular}{lccc}
\hline \multicolumn{1}{c}{ Covariates } & \multicolumn{2}{c}{ HR estimate } & $\boldsymbol{P}$ \\
\cline { 2 - 3 } & Point & $\mathbf{9 5 \%} \mathbf{C I}$ & value \\
\hline Entire cohort $(\mathrm{N}=347)$ & & & \\
$\quad$ SLR vs lobectomy & & & \\
$\quad$ Adjusted by propensity score & 1.3 & $(0.5-3.3)$ & .62 \\
$\quad$ Stratified by propensity score quintiles & 1.1 & $(0.4-2.9)$ & .79 \\
Cohort $\leq 2 \mathrm{~cm}(\mathrm{~N}=306)$ & & & \\
$\quad$ SLR vs lobectomy & & & \\
$\quad$ Adjusted by propensity score & 1.5 & $(0.6-4.2)$ & .42 \\
$\quad$ Stratified by propensity score quintiles & 1.4 & $(0.5-3.8)$ & .52 \\
Secondary analysis (N = 331) & & & \\
$\quad$ Wedge resection vs lobectomy & & & \\
$\quad$ Adjusted by propensity score & 1.9 & $(0.7-5.0)$ & .19 \\
$\quad$ Stratified by propensity score quintiles & 2.0 & $(0.7-5.3)$ & .17 \\
\hline
\end{tabular}

$C I$, Confidence interval; $H R$, hazard ratio; $S L R$, sublobar resection. solid nodule, when controlling for potential confounders. The tightly controlled prospective acquisition of data elements, including comorbidities, presence of emphysema, and $\mathrm{CAC}$ on the baseline CT scan and the use of the propensity scoring to create a balance of the covariates between the 2 surgical treatments, supports the validity of these findings. Another important finding is the remarkably low surgical mortality $(0.9 \%)$ in this screen-detected cohort treated by general thoracic surgeons at centers with specialized screening programs. We believe that the low operative mortality in this report and that reported in the American College of Surgeons Oncology Group Z030 trial ${ }^{30}$ are more representative of the current state of the art rather than the $5 \%$ mortality frequently cited in the literature.

Our results are similar to those previously reported in a study using the National Cancer Institute-sponsored registry that included 1165 patients, of whom 969 were treated by LR and 196 were treated by SLR. ${ }^{13}$ In that study, survival of patients with stage IA tumors $20 \mathrm{~mm}$ or less was similar for both treatment modalities. Notwithstanding our data and those of others supporting SLR, there are a number of reports that assert that SLR is associated with higher local recurrence rates and inferior disease-free survival and should be reserved for patients at high risk for LR. ${ }^{31,32} \mathrm{~A}$ recent meta-analysis comparing LR with SLR performed in the 1990s and early 2000 showed a statistically insignificant survival advantage of LR. ${ }^{33}$ However, there was considerable heterogeneity among studies in both patient selection criteria and tumor characteristics. Most studies 


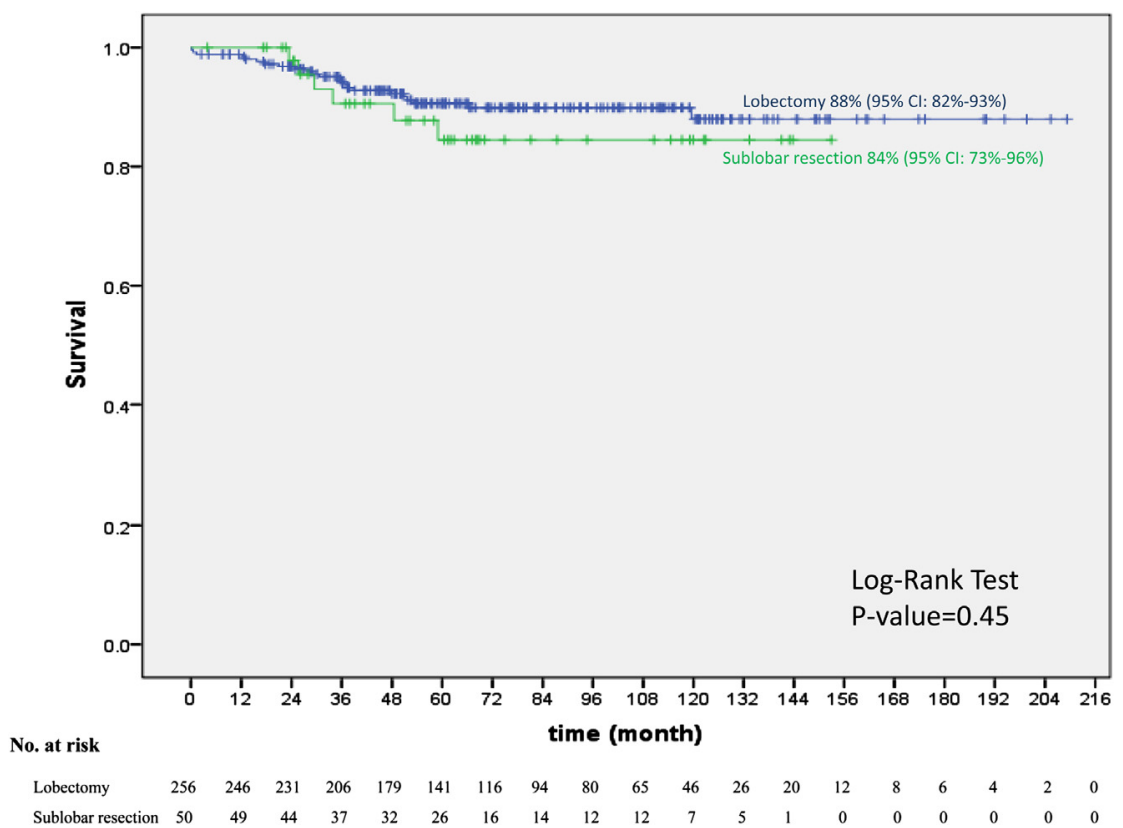

FIGURE 2. Kaplan-Meier survival curves for 306 patients with clinical stage IA lung cancer manifesting as a solid nodule (nodule diameter of $\leq 20 \mathrm{~mm}$ ), separately for those who underwent lobectomy $(\mathrm{n}=256)$ and SLR $(\mathrm{n}=50) . C I$, Confidence interval.

combined wedge resection and segmentectomy, which are generally not regarded by most thoracic surgeons as oncologically equivalent operations. As video-assisted thoracoscopic surgery becomes the state-of-the-art practice for surgery in the context of screening, these issues will continue to be revisited. ${ }^{34}$

The assignment of patients in this study to SLR or LR clearly was not randomized; therefore, the possibility that patients with limited pulmonary reserve were not selected for LR cannot be excluded. However, the use of propensity scoring provides a methodology for correcting for this potential recruitment bias because it mimics randomization by creating comparable samples of individuals who underwent SLR and LR on the basis of the covariates. The presence of emphysema on the baseline CT scan at the time of enrollment is a reasonable surrogate of pulmonary function and has been shown to be a significant predictor of death from chronic obstructive pulmonary disease and lung cancer. ${ }^{17-21}$ In addition, the proportion of patients with marked emphysema and CAC score were not significantly different between the 2 treatment groups. Finally, because only a small proportion of patients in the SLR group had tumors larger than $2 \mathrm{~cm}$, our conclusions cannot be extended with a high degree of confidence to the treatment of patients with $\mathrm{T} 1 \mathrm{~b}$ disease.

An interesting observation is that even in the hands of qualified thoracic surgeons, $21 \%$ and $43 \%$ of patients treated by LR and SLR, respectively, did not have any mediastinal nodal sampling. This imbalance in surgical mediastinal staging may have adversely affected the outcome after
SLR because the LR group would have been more thoroughly staged. However, despite this imbalance, there was no difference in survival between the 2 groups. Clearly, regardless of the extent of parenchymal resection, appropriate staging of the mediastinum should be performed whenever possible because it has important therapeutic and prognostic implications. Another important surgical question is the frequency of local and loco-regional recurrence between the 2 treatment groups and between the 2 forms of SLR. Several studies, including the randomized trial by the LCSG, showed that SLR was associated with a significantly higher incidence of local recurrence and a $50 \%$ increase in cancer-related deaths. ${ }^{1,4}$ Others have suggested that in appropriately selected patients, local failure rates were similar regardless of the extent of parenchymal resection. ${ }^{7,8}$ There are important differences between these studies in patient selection criteria and the type of SLR performed, particularly because wedge resections are reportedly associated with a significantly higher local recurrence rate compared with anatomic segmentectomy. ${ }^{35}$ We found no significant difference between LR and wedge SLR, but segmentectomy had a lower recurrence rate than wedge SLR, although the small sample size of the SLR group somewhat limits this result. Planned future studies and the ongoing randomized trials should provide the answers to these critical questions.

\section{CONCLUSIONS}

To our knowledge, this is the first report on the merits of SLR in the context of screening, and it focuses only on lung 
cancers manifesting as solid nodules. The finding that LR and SLR are associated with similar lung cancer-specific survival in screen-detected cancers will add to the treatment options for patients with early-stage lung cancer as wider implementation of screening develops. The important debate about the role of SLR in the treatment of earlystage lung cancer will ultimately be resolved by the ongoing adequately powered randomized trials conducted in North America by the Alliance of Clinical trials in Oncology (Cancer and Leukemia Group B 140503) and in Japan by the Japanese Clinical Oncology Group.

\section{References}

1. Ginsberg RJ, Rubinstein LV. Randomized trial of lobectomy versus limited resection for T1 N0 non-small-cell lung cancer. Lung Cancer Study Group. Ann Thorac Surg. 1995;60:615-22.

2. Read RC, Yoder G, Schaeffer RC. Survival after conservative resection for T1 N0 M0 non-small cell lung cancer. Ann Thorac Surg. 1990;49:391-8.

3. Warren WH, Faber LP. Segmentectomy versus lobectomy in patients with stage I pulmonary carcinoma. Five-year survival and patterns of intrathoracic recurrence. J Thorac Cardiovasc Surg. 1994;107:1087-93.

4. Landreneau RJ, Sugarbaker DJ, Mack MJ, Hazelrigg SR, Luketich JD, Fetterman L, et al. Wedge resection versus lobectomy for stage I (T1 N0 M0) non-small-cell lung cancer. J Thorac Cardiovasc Surg. 1997;113:691-8.

5. Tsubota N, Ayabe K, Doi O, Mori T, Namikawa S, Taki T, et al. Ongoing prospective study of segmentectomy for small lung tumors. Study Group of Extended Segmentectomy for Small Lung Tumor. Ann Thorac Surg. 1998;66: 1787-90.

6. Pastorino U, Valente M, Bedini V, Infante M, Tavecchio L, Ravasi G. Limited resection for Stage I lung cancer. Eur J Surg Oncol. 1991;17:42-6.

7. Kodama K, Doi O, Higashiyama M, Yokouchi H. Intentional limited resection for selected patients with T1 N0 M0 non-small cell lung cancer: a singleinstitution study. J Thorac Cardiovasc Surg. 1997:114:347-53.

8. Koike T, Yamato Y, Yoshiya K, Shimoyama T, Suzuki R. Intentional limited pulmonary resection for peripheral T1 N0 M0 small-sized lung cancer. J Thorac Cardiovasc Surg. 2003;125:924-8.

9. Keenan RJ, Landreneau RJ, Maley RH Jr, Singh D, Macherey R, Bartley S, et al Segmental resection spares pulmonary function in patients with stage I lung cancer. Ann Thorac Surg. 2004;78:228-33.

10. Harada H, Okada M, Sakamoto T, Matsuoka H, Tsubota N. Functional advantage after radical segmentectomy versus lobectomy for lung cancer. Ann Thorac Surg. 2005;80:2041-5.

11. Okada M, Nishio W, Sakamoto T, Uchino K, Yuki T, Nakagawa A, et al. Effect of tumor size on prognosis in patients with non-small cell lung cancer: the role of segmentectomy as a type of lesser resection. J Thorac Cardiovasc Surg. 2005; 129:87-93.

12. Yoshida J, Nagai K, Yokose T, Nishimura M, Kakinuma R, Ohmatsu H, et al. Limited resection trial for pulmonary ground-glass opacity nodules: fifty-case experience. J Thorac Cardiovasc Surg. 2005;129:991-6.

13. Wisnivesky JP, Henschke CI, Swanson S, Yankelevitz DF, Zulueta J, Marcus S, et al. Limited resection for the treatment of patients with stage IA lung cancer. Ann Surg. 2010;251:550-4.

14. Kates M, Swanson S, Wisnivesky JP. Survival following lobectomy and limited resection for the treatment of stage I non-small-cell lung cancer $<1 \mathrm{~cm}$ in size: a review of SEER data. Chest. 2011;139:491-6.

15. Henschke CI, Yankelevitz DF, Smith JP, Miettinen OS. Screening for lung cancer: the early lung cancer action approach. Lung Cancer. 2002;35:143-8.

16. International Early Lung Cancer Action Program protocol. Available at: www. IELCAP.org/professionals/docs/ielcap.pdf. Accessed April 4, 2013.

17. Zulueta JJ, Wisnivesky JP, Henschke CI, Yip R, Farooqi AO, McCauley DI, et al. Emphysema scores predict death from COPD and lung cancer. Chest. 2012;141: 1216-23.

18. Shemesh J, Henschke CI, Shaham D, Yip R, Farooqi AO, Cham ID, et al. Ordinal scoring of coronary artery calcifications on low-dose CT scans of the chest is predictive of death from cardiovascular disease. Radiology. 2010;257:541-8.

19. De Torres JP, Bastarrika G, Wisnivesky JP, Alcaide AB, Campo A, Seijo LM, et al. Assessing the relationship between lung cancer risk and emphysema detected on low-dose CT of the chest. Chest. 2007;132:1932-8.
20. Wilson DO, Weissfeld JL, Balkan A, Schragin JG, Fuhrman CR, Fisher SN, et al Association of radiographic emphysema and airflow obstruction with lung cancer. Am J Respir Crit Care Med. 2008;178:738-44.

21. Ueda K, Jinbo M, Li TS, Yagi T, Suga K, Hamano K. Computed tomographydiagnosed emphysema, not airway obstruction, is associated with the prognostic outcome of early-stage lung cancer. Clin Cancer Res. 2006;12:6730-6.

22. Vazquez M, Flieder D, Travis W, Carter D, Yankelevitz DF, Miettinen OS, et al Early lung cancer action project pathology protocol. Lung Cancer. 2003;39: 231-2.

23. Carter D, Vazquez M, Flieder DB, Brambilla E, Gazdar A, Noguchi M, et al Comparison of pathologic findings of baseline and annual repeat cancers diagnosed on CT screening. Lung Cancer. 2007;56:193-9.

24. Vazquez M, Carter D, Brambilla E, Gazdar A, Noguchi M, Travis WD, et al. Sol itary and multiple resected adenocarcinomas after CT screening for lung cancer: histopathologic features and their prognostic implications. Lung Cancer. 2009 64:148-54.

25. Travis WD, Brambilla E, Muller-Hermelink HK, Harris CC. Pathology and Genetics: Tumours of the Lung, Pleura, Thymus and Heart. Lyon, France: IARC; 2004.

26. Travis WD, Brambilla E, Noguchi M, Nicholson AG, Geisinger KR, Yatabe Y, et al. International Association for the Study of Lung Cancer/American Thoracic Society/European Respiratory Society International Multidisciplinary Classification of Lung Adenocarcinoma. J Thorac Oncol. 2011;6:244-85.

27. International Early Lung Cancer Action Program Investigators. Survival of patients with stage I lung cancer detected on CT screening. N Engl J Med. 2006 355:1763-71.

28. National Lung Screening Trial Research Team. Reduced lung-cancer mortality with low-dose computed tomographic screening. $N$ Engl J Med. 2011;365: 395-409.

29. Dell'Amore A, Monteverde M, Martucci N, Sanna S, Caroli G, Stella F, et al Early and long-term results of pulmonary resection for non-small-cell lung cancer in patients over 75 years of age: a multi-institutional study. Interact Cardiovasc Thorac Surg. 2013;16:250-6.

30. Allen MS, Darling GE, Pechet TT, Mitchell JD, Herndon JE 2nd, Landreneau RJ, et al., ACOSOG Z0030 Study Group. Morbidity and mortality of major pulmonary resections in patients with early-stage lung cancer: initial results of the randomized, prospective ACOSOG Z0030 trial. Ann Thorac Surg. 2006;81:1013-9.

31. Mery CM, Pappas AN, Bueno R, Colson YL, Linden P, Sugarbaker DJ, et al Similar long-term survival of elderly patients with non-small cell lung cancer treated with lobectomy or wedge resection within the Surveillance, Epidemiology, and End Results database. Chest. 2005;128:237-45.

32. Rami-Porta R, Tsuboi M. Sublobar resection for lung cancer. Eur Respir J. 2009 33:426-35.

33. Nakamura H, Kawasaki N, Taguchi M, Kabasawa K. Survival following lobectomy vs limited resection for stage I lung cancer: a meta-analysis. Br J Cancer. 2005;92:1033-7.

34. Petersen RH, Hansen HJ, Dirksen A, Pedersen JH. Lung cancer screening and video-assisted thoracic surgery. J Thorac Oncol. 2012;7:1026-31.

35. Sienel W, Stremmel C, Kirschbaum A, Hinterberger L, Stoelben E, Hasse J, et al Frequency of local recurrence following segmentectomy of stage IA non-small cell lung cancer is influenced by segment localisation and width of resection margins-implications for patient selection for segmentectomy. Eur J Cardiothorac Surg. 2007;31:522-7

\section{APPENDIX 1. THE I-ELCAP INVESTIGATORS}

Mount Sinai School of Medicine, New York, NY: Claudia I. Henschke, Principal Investigator, David F. Yankelevitz, Rowena Yip, Dongming Xu, David S. Mendelson; McGill University: Olli S. Miettinen; Weill Cornell Medical College: Dorothy I. McCauley, Mildred Chen, Daniel M. Libby, Olli S. Miettinen, James P. Smith, Mark Pasmantier; Cornell University: A. P. Reeves, A. Biancardi; CBNS, City University of New York at Queens College, Queens, NY: Steven Markowitz, Albert Miller; Azumi General Hospital, Nagano, Japan: Shusuke Sone, Takaomi Hanaoka; University of Toronto, Princess Margaret Hospital, Toronto, 
Canada: Heidi Roberts, Demetris Patsios; Clinica Universitaria de Navarra, Pamplona, Spain: Javier Zulueta, Luis Montuenga, Maria D. Lozano; Christiana Care, Helen F. Graham Cancer Center, Newark, Del: Thomas Bauer; National Cancer Institute Regina Elena, Rome, Italy: Salvatore Giunta; LungenZentrum Hirslanden, Zurich, Switzerland: Karl Klingler; Swedish Medical Center, Seattle, Wash: Ralph Aye; Columbia University Medical Center, New York, NY: John H. M. Austin, Gregory D. N. Pearson; Hadassah Medical Organization, Jerusalem, Israel: Dorith Shaham; St Agnes Cancer Center, Baltimore, Md: Enser Cole; New York University Medical Center, New York, NY: David Naidich, Georgeann McGuinness; Holy Cross Hospital Cancer Institute, Silver Spring, Md: Cheryl Aylesworth; State University of New York at Stony Brook, Stony Brook, NY: Matthew Rifkin; Maimonides Medical Center, Brooklyn, NY: Samuel Kopel; Roswell Park Cancer Institute, Buffalo, NY: Donald Klippenstein, Alan Litwin, Peter A. Loud; State University of New York, Upstate Medical Center, Syracuse, NY: Leslie J. Kohman, Ernest M. Scalzetti; Dorothy E. Schneider Cancer Center, MillsPeninsula Health Services, San Mateo, Calif: Barry Sheppard; ProHealth Care Regional Cancer Center, Waukesha and Oconomowoc Memorial Hospitals, Oconomowoc, Wis: M. Kristin Thorsen, Richard Hansen; North ShoreLong Island Jewish Health System, New Hyde Park, NY: Arfa Khan, Rakesh Shah; Jackson Memorial Hospital, University of Miami, Miami, Fla: Richard Thurer; Eisenhower Lucy Curci Cancer Center, Rancho Mirage, Calif; Davood Vafai; The 5th Affiliated Hospital of Sun Yat-Sen University, Zhuhai, China: Xueguo Liu; South Nassau Communities Hospital, Long Island, NY: Shahriyour Andaz; Fundacion Instituto Valenciano de Oncologia, Valencia, Spain: Jose Cervera Deval; Georgia Institute for Lung Cancer Research, Atlanta, Ga: Michael V. Smith; Nebraska Methodist Hospital, Omaha, Neb: Patrick Meyers; Shin Kong Wu Ho-Su Memorial Hospital, Taipei, Taiwan: Diane Yeh; St Joseph Health Center, St Charles, Mo: Dan Luedke; Memorial Sloan-Kettering Cancer Center, New York, NY: Robert T. Heelan, Michelle S. Ginsberg; New York Medical College, Valhalla, NY: Terence A. S. Matalon; Mount Sinai Comprehensive Cancer Center, Miami Beach, Fla: Shari-Lynn Odzer; Wellstar Health System, Marietta Ga: William Mayfield; City of Hope National Medical Center, Duarte, Calif: Fred Grannis, Arnold Rotter; Evanston Northwestern Healthcare Medical Group, Evanston, Ill: Daniel Ray; Aurora St Luke's Medical Center, Milwaukee Wis: David Olsen; Staten Island University Hospital, Staten Island, NY: Mary Salvatore; Our Lady of Mercy Medical Center, Bronx, NY: Peter H. Wiernik; The Valley Hospital Cancer Center, Paramus, NJ: Robert Korst; Greenwich Hospital, Greenwich, Conn: David Mullen; Glens Falls Hospital, Glens Falls, NY: Louis DeCunzo; Karmanos Cancer Institute, Detroit, Mich: Harvey
Pass, Carmen Endress; Sharp Memorial Hospital, San Diego, Calif: Michael Kalafer; John Muir Cancer Institute, Concord, Calif: Michaela Straznicka; Sequoia Hospital, Redwood City, Calif: Melissa Lim; Alta Bates Summit Medical Center, Berkeley, Calif: Gary Cecchi; Bend Memorial Hospital, Bend, Ore: Albert Koch; St Joseph's Hospital, Atlanta, Ga: Paul Scheinberg; Baylor University Medical Center, Dallas Tex: Edson H. Cheung.

\section{Discussion}

Dr Joseph Friedberg (Philadelphia, $\mathrm{Pa}$ ). The role of SLRs has been the focus of intense scrutiny by thoracic surgeons for decades, and the exact role has yet to be rigorously defined. We are still forced to fall back on the LCSG analysis from 1995 as the final word until the upcoming randomized trials from the Cancer and Leukemia Group B and the Japanese Clinical Oncology Group are completed. Your study provides more compelling evidence, however, that there is clearly a role for SLR in some of our patients.

My first question relates to the study population. As I read the article, I was struck at how astoundingly well matched the 2 groups were in this study, right down to the percentage with college educations. I believe this is a particular strength of this study in that it allows for an accurate assessment of outcomes attributable to the surgical procedure. A potential weakness of this study, however, arises from this same degree of uniformity. Specifically, this study is based on a subgroup of a subgroup - a group of patients who qualified for and enrolled in a lung cancer screening trial and then developed solid nodules while in that trial. Do you offer any cautions or see any reasons why your results would not apply to the more general population of patients with lung cancer we see in our practices?

Dr Altorki. I don't believe this study is a practice-changing study. However, it is a study that calls for more equipoise in terms of our approach to the treatment of lung cancer using limited resections. We have applied these operations in patients who would be candidates for both lobectomy and SLR. Patients who are poor candidates for lobectomy should be treated by SLR.

Dr Friedberg. My second question centers on another unusual situation for the general population of patients with lung cancer. All of these patients had their surgery performed by general thoracic surgeons, not just general thoracic surgeons, but highly qualified general thoracic surgeons. Within this context, I was surprised that $70 \%$ of the SLRs were performed as wedges, not anatomic segmentectomies. I was also surprised that more than $40 \%$ of the patients undergoing SLR and approximately one quarter of the patients undergoing lobectomy did not have even 1 mediastinal lymph node biopsied. One would expect, on the basis of the LCSG analysis and common sense, that some of these patients, especially those undergoing SLR, were understaged or undertreated, yet the results are as good as anything in the literature. How do you reconcile that? 
Dr Altorki. Let me take the second part first. I do agree with you that the assessment of the mediastinal nodes was disappointing. However, it exceeds what is in the published literature. We need to further educate about the therapeutic and prognostic impact of mediastinal nodal staging, particularly in patients in whom an SLR is performed. My guess is that most of these patients underwent video-assisted thoracic surgery, and, as you know, mediastinal nodal assessment by video-assisted thoracic surgery may not be as straightforward as it is by the open procedure, so that's probably why they were skipped.

To go back to the wedge resection, many may have thought going into this that a $1-\mathrm{cm}$ or $1.2-\mathrm{cm}$ tumor may be effectively treated by wedge resection, and that is the job we have to address in the ongoing randomized trials to try and clarify if there is indeed a role for wedge resection.

Dr Friedberg. I am curious about the decision to omit patients with ground-glass opacities from the study. I suspect many of us, given the controversy surrounding SLR, are more likely to be aggressive with using SLRs for a slowly progressing ground-glass opacity than a solid nodule. Do you know how many patients were excluded from the study because their nodules weren't solid and do you have any thoughts on the applicability of SLRs for these patients?

Dr Altorki. We had this discussion, and I voted strongly to exclude them. I do believe that they are a group of patients who exhibit a different biological behavior than those with solid nodules, and we wanted to focus on a specific group of patients. But I agree with you that this is a group that needs to be studied and we hope to report on.

Dr Friedberg. They are the ones who are more likely to develop something down the road requiring additional surgery. It seems to me they would be the best candidates for this.

Dr Altorki. I think they are good candidates for SLR, yes, but I do think they have to be studied separately.

Dr Scott Swanson (Boston, Mass). I like this study, Nasser. I was struck by the $5 \%$ of second tumors in the lobectomy specimen. Presumably it is the same in the wedge, but the local recurrence rates weren't different. Do you have any data about little nodules that were being followed in that wedge group and what happened or why that is?

Dr Altorki. That number is consistent with the published literature from Japan specifically and from our institution outside of the I-ELCAP. These are usually not detected clinically on the CT scans. We do have some patients in the Cancer and Leukemia Group B trial in whom a wedge resection was performed, and even in the wedge, there are additional nodules. The bottom line is that it did not affect lung cancer-specific survival. But I'm concerned about them, as you are.

Dr L. Penfield Faber (Chicago, Ill). Dr Altorki, I compliment you on an excellent presentation. I would also like to thank my good friends, Drs Thurer, Kohman, and Harvey Pass, for substantiating the surgical concept of surgical resection for primary lung cancer that Dr Jensik and I reported on 34 years ago, both qualified general thoracic surgeons. We described 168 patients with a $53 \%$ 5 -year survival. We resected larger tumors, greater than $3 \mathrm{~cm}$, and our lymph node staging was inadequate, and I think that accounts for our low survival compared with what we see today. With the guidelines reported today, I believe segmental resection for primary lung cancer is a good operation.

Dr Altorki. I have to acknowledge the pioneering contribution of Dr Faber and, as a displaced Chicagoan, of Dr Jensik as well. So I thank you for your efforts.

Dr Jose Rodriguez (Dayton, Ohio). I noticed that in your 51 or 52 SLRs, there were maybe 18 that were segmentectomies. It was not exactly described whether they were anatomic or nonanatomic. Do you think that it is a low number of segmentectomies to compare with approximately 200 lobectomies? Do you think that it is a low-power study to say that a segmentectomy might be better?

I noticed that $4 \%$ to $6 \%$ of your SLRs were upstaged to stage II or III because of N1 or N2 unexpected disease. What did you do with those patients? Now you need to give adjuvant treatment, and, in my opinion, they received maybe a suboptimal resection for a stage II or III. I would be concerned that we are advising surgeons to do an SLR when we are going to have maybe 5\% to $7 \%$ or $10 \%$ of patients who will have a suboptimal resection due to unexpected stage II and III. I would like you to comment on that.

Dr Altorki. We are not advising the surgeon to do anything. We are just advising the surgeon to develop some equipoise. We advise the surgeon that the data from the LCSG are no longer relevant and that you should approach this whole matter with a new frame of mind. We need to do a study that compares one with the other.

Dr Daniel Miller (Atlanta, $\mathrm{Ga}$ ). Did you go back and look at all and see if there was a difference in the pathologic upstaging in regard to a nodule that was found on the incident scan versus the prevalent scan? Many times when we have found these original nodules, they are a bit more biologically active, they might already have nodal involvement and so forth. It would be an interesting question. As we look at the follow-up scans, those might be better survival and so forth and be something to look at because you have a larger number of patients.

Dr Altorki. You are saying segregate the cancers on the baseline from the cancers on the annual repeat?

Dr Miller. Exactly, yes.

Dr Altorki. That information has been published by I-ELCAP before. Conceptually, though, we do think those that occur on the annual repeat are more likely to be the more aggressive ones than those detected on the baseline scans. I agree with you.

Dr Caio Sterse Da Mata (Sao Paulo, Brazil). What was the criteria you used to perform a wedge versus a segmentectomy?

Dr Altorki. These patients were involved in the local screening program, and the management was left to the local tumor committees of the local hospitals. We did not mandate any type of resection or how the resection should be done.

Dr Da Mata. There was no inclusion or exclusion criteria for this?

Dr Altorki. No. The local surgeon, who was usually a boardcertified or equivalent certified thoracic surgeon, made the decision about that.

Dr Frank Detterbeck (New Haven, Conn). Dan Miller is always a step ahead of me and asked more or less the question I wanted to ask. 
I have 2 follow-up points. You can look at your data to find out how many of these were baseline versus follow-up scans and do that analysis, and I would stimulate you to perhaps ask the question: In your practice, because you are in an area where there is a fair amount of screening performed, but there is also a fair amount of incidental scanning going on, can you look at whether incidental scanning gives us similar results to screening? That would have a lot of impact in the generalizability of the results that you have just reported.

Dr Altorki. The crux of the question is that these were patients in a screening trial, and therefore they were a special cohort. I accept that, and we don't say that in our summary statement.
Does that translate into what you find in somebody who comes to your office with a CT scan in hand? I think to answer that question we have an ongoing randomized trial.

Dr Joseph Shrager (Stanford, Calif). I want to go back to the issue of the tumor formerly known as bronchioloalveolar carcinoma. You said solid nodules, but does that mean part-solid nodules? You don't have any nodules in there that are half ground-glass or anything like that?

Dr Altorki. No. We excluded all part-solid and nonsolid nodules from this analysis.

Dr Shrager. Okay. I think that's a really important point.

Dr Altorki. I agree with you, yes. 